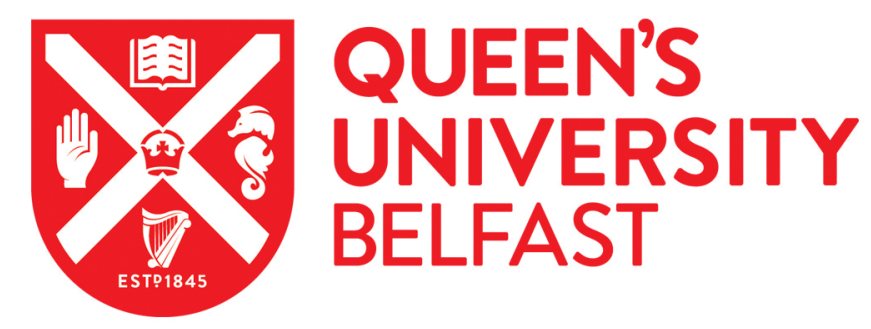

\title{
Arbitrary amplitude ion-acoustic solitary excitations in the presence of excess superthermal electrons
}

Saini, N., Kourakis, Y., \& Hellberg, M. A. (2009). Arbitrary amplitude ion-acoustic solitary excitations in the presence of excess superthermal electrons. Physics of Plasmas, 16(6), [062903].

https://doi.org/10.1063/1.3143036

Published in:

Physics of Plasmas

Queen's University Belfast - Research Portal:

Link to publication record in Queen's University Belfast Research Portal

\section{General rights}

Copyright for the publications made accessible via the Queen's University Belfast Research Portal is retained by the author(s) and / or other copyright owners and it is a condition of accessing these publications that users recognise and abide by the legal requirements associated with these rights.

Take down policy

The Research Portal is Queen's institutional repository that provides access to Queen's research output. Every effort has been made to ensure that content in the Research Portal does not infringe any person's rights, or applicable UK laws. If you discover content in the Research Portal that you believe breaches copyright or violates any law, please contact openaccess@qub.ac.uk. 


\title{
Arbitrary amplitude ion-acoustic solitary excitations in the presence of excess superthermal electrons
}

\author{
N. S. Saini, ${ }^{1, a)}$ I. Kourakis, ${ }^{1, b)}$ and M. A. Hellberg ${ }^{2, c)}$ \\ ${ }^{1}$ Centre for Plasma Physics, Department of Physics and Astronomy, Queen's University Belfast, \\ Belfast BT7 1NN, Northern Ireland, United Kingdom \\ ${ }^{2}$ School of Physics, University of KwaZulu-Natal, Private Bag X54001, Durban 4000, South Africa
}

(Received 13 March 2009; accepted 4 May 2009; published online 9 June 2009)

\begin{abstract}
Velocity distribution functions with an excess of superthermal particles are commonly observed in space plasmas, and are effectively modeled by a kappa distribution. They are also found in some laboratory experiments. In this paper we obtain existence conditions for and some characteristics of ion-acoustic solitary waves in a plasma composed of cold ions and $\kappa$-distributed electrons, where $\kappa>3 / 2$ represents the spectral index. As is the case for the usual Maxwell-Boltzmann electrons, only positive potential solitons are found, and, as expected, in the limit of large $\kappa$ one recovers the usual range of possible soliton Mach numbers, viz., $1<M<1.58$. For lower values of $\kappa$, modeling the presence of a greater superthermal component, the range of accessible Mach numbers is reduced. It is found that the amplitude of the largest possible solitons that may be generated in a given plasma (corresponding to the highest allowed Mach number for the given plasma composition) falls off with decreasing $\kappa$, i.e., an increasing superthermal component. On the other hand, at fixed Mach number, both soliton amplitude and profile steepness increase as $\kappa$ is decreased. These changes are seen to be important particularly for $\kappa<4$, i.e., when the electrons have a "hard" spectrum. (C) 2009 American Institute of Physics. [DOI: 10.1063/1.3143036]
\end{abstract}

\section{INTRODUCTION}

The scope of the article at hand embraces the nonlinear dynamics of ion-acoustic (IA) waves under the effect of a non-Maxwellian electron velocity distribution with excess superthermal particles, represented by a $\kappa$ distribution. The basic prerequisites of our study are outlined in the following paragraphs.

Plasmas are often characterized by a particle distribution function with a high energy tail and they may thus deviate significantly from a Maxwellian. ${ }^{1-3}$ Both space and laboratory plasma environments may have such an excess superthermal electron population due to velocity space diffusion, which may lead to an inverse power-law distribution at a velocity much higher than the electron thermal speed. ${ }^{4-6}$ Such behavior is effectively modeled by a kappa (or generalized Lorentzian) distribution function, ${ }^{1,6-8}$ which appears to be more appropriate than a thermal (Maxwellian) distribution in a wide range of plasma situations.

The commonly used three-dimensional, isotropic kappa ( $\kappa)$ distribution is given by ${ }^{6,8}$

$$
f_{\kappa}(v)=\frac{n_{0}}{\left(\pi \kappa \theta^{2}\right)^{3 / 2}} \frac{\Gamma(\kappa+1)}{\Gamma\left(\kappa-\frac{1}{2}\right)}\left(1+\frac{v^{2}}{\kappa \theta^{2}}\right)^{-(\kappa+1)},
$$

where $n_{0}$ is the species equilibrium number density, $\theta^{2}=[(\kappa$ $-3 / 2) / \kappa]\left(2 k_{B} T / m\right)$ is the effective thermal speed, modified by the spectral index $\kappa$, with $T$ the kinetic temperature and $m$ the species mass, and $\Gamma(x)$ is the gamma function. Here $v^{2}$ $=v_{x}^{2}+v_{y}^{2}+v_{z}^{2}$ obviously denotes the square norm of the veloc-

\footnotetext{
a) Electronic mail: nssaini@yahoo.com and ns.saini@qub.ac.uk.

${ }^{b)}$ Electronic mail: i.kourakis@qub.ac.uk.

${ }^{c)}$ Electronic mail: hellberg@ukzn.ac.za.
}

ity $\mathbf{v}$. Clearly, for a physically realistic thermal speed, one requires $\kappa>3 / 2$. At very large values of the spectral index $\kappa$, the velocity distribution function approaches a Maxwellian distribution. Low values of $\kappa$ represent distributions with a relatively large component of particles with speed greater than the thermal speed ("superthermal particles") and an associated reduction in "thermal" particles, as one observes in a "hard" spectrum.

First applied by Vasyliunas ${ }^{1}$ to model observations of particle energy distributions in space-based experiments, the $\kappa$ distribution is widely used to fit velocity distributions observed in space plasmas, often with $2<\kappa<6$. Examples include measurements of plasma sheet electron and ion distributions $\left(\kappa_{i}=4.7\right.$ and $\left.\kappa_{e}=5.5\right),{ }^{9}$ and observations in the earth's foreshock $\left(3<\kappa_{e}<6\right) .{ }^{10}$ Modelers have also used $\kappa$ distributions with low values of $\kappa$, e.g., Pierrard and co-workers ${ }^{11,12}$ developed a Lorentzian ion exosphere model and associated solar wind model with coronal electrons satisfying $2<\kappa_{e}$ $<6$. Although there is no completely satisfactory theory for the persistence and apparent ubiquity of $\kappa$ distributions in space, works by Treumann and co-workers, ${ }^{13,14}$ Leubner, ${ }^{4}$ and Collier, ${ }^{15}$ provided heuristic explanations or pointers toward a full explanation.

It has been argued that a combination of kappa distributions models multicomponent plasmas more effectively than a superposition of Maxwellians. ${ }^{16,17}$ Indeed, recent observations of the electron velocity distribution function in Saturn's magnetosphere appear to confirm this view. ${ }^{5}$

By integrating the kappa distribution function over velocity space, one can obtain the number density of the corresponding plasma constituent(s), which affects the charge balance via Poisson's equation. An important characteristic 
of the kappa distribution function is that the dependence of the density on the electrostatic potential differs from the familiar exponential form obtained from the MaxwellBoltzmann distribution. The consequences will be obvious in our analysis below, both from an analytical and a numerical point of view.

The linear properties of plasmas in the presence of a kappa distribution with excess superthermal particles have been investigated rather extensively. A modified plasma dispersion function for such a "superthermal" plasma was introduced in Ref. 16 for integer $\kappa$, and was extended to a generalized plasma dispersion function for arbitrary real $\kappa^{18,19}$ The usual plasma dispersion function (derived for the Maxwellian case) is obtained by both these approaches in the limit of infinite $\kappa$, as expected. It has been shown ${ }^{20}$ that the generalized plasma dispersion function, $Z_{\kappa}$ could provide a plasma diagnostic in space, in that wave data recorded in the magnetosphere could be used to find the appropriate $\kappa$ value characterizing the electron distribution function, clearly differentiating it from results calculated using a Maxwellian assumption. Similarly, it was found that wave experiments can act as a diagnostic for the distribution function in a laboratory plasma when use is made of the $Z_{\kappa}$ plasma dispersion function. ${ }^{6}$

The effect of superthermal electrons on linear IA waves propagating in a magnetized plasma was studied in Ref. 19 Interestingly, the presence of a high energy tail leads to a significant variation in the damping rate of electrostatic plasma waves, as compared to Maxwellian plasmas, ${ }^{21}$ so excess superthermality was found in that case to enhance Landau damping.

The founding blocks of a nonlinear theory for IA plasma excitations were provided four decades ago, with a study of small-amplitude nonlinear excitations, ${ }^{22}$ and an arbitrary amplitude theory for IA solitary waves. ${ }^{23,24}$ A model was proposed to study the dynamics of solitary waves in an electronion plasma, ${ }^{23}$ and a domain for the Mach number ( $M$ $\in[1,1.58])$ was found for the existence of solitary waves. The pseudopotential method developed by Sagdeev ${ }^{23}$ for nonlinear IA excitations (later extended to describe magnetized plasmas ${ }^{25}$ ) predicted that only positive potential disturbances may occur in simple electron-ion plasmas. Nevertheless, negative potential solitary structures have later been shown to exist in the presence of two electron populations, ${ }^{26,27}$ and/or in multi-ion plasma compositions or dusty plasmas. ${ }^{28-31}$

It may be added for completeness that another approach to velocity space nonthermality is provided by the so called Tsallis distribution. ${ }^{32}$ Like the kappa distribution, the Tsallis distribution represents a family of distribution functions, governed by a single parameter ( $q$, in this case), and possessing a power-law structure (the power is given by $1 /[1-q]$ ), with the Maxwellian as a limiting case, when $q \rightarrow 1$. Unfortunately, although there are similarities, there is no simple transformation between the Tsallis and kappa distribution (1), as the forms of the argument and the power do not both fit the same transformation. However, one may wish to use an approximate relationship given by $\kappa \rightarrow 1 /(q-1)$, in that, for $q>1$, an increase in $q$ increases the fraction of superther-

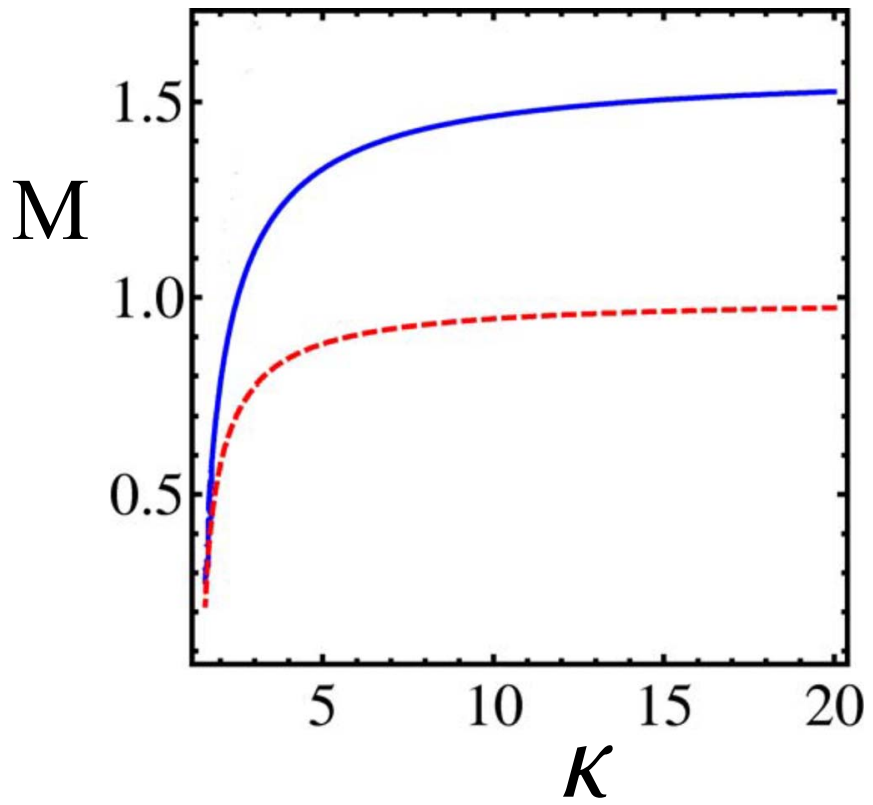

FIG. 1. (Color online) IA soliton existence domain in the parameter space of $\kappa$ and Mach number, $M$. Solitons may be supported in the region between the two curves. The lower, dashed curve represents the minimum (soliton) condition, $M_{1}$, and the upper, solid curve the infinite compression limit, $M_{2}$.

mal electrons relative to that of the Maxwellian, which is equivalent to a decrease in $\kappa$. Recently, existence conditions have been found for IA solitons in a plasma composed of cold ions and electrons modeled by a Tsallis distribution. ${ }^{33}$ The main results were that (i) as for the conventional IA solitons based on a Maxwellian distribution, only positive solitons were found, and (ii) the accessible range in Mach number found for a Maxwellian, [1,1.58], is reduced as $q$ is increased beyond $q=1$, i.e., increasing the superthermal excess reduces the range of propagation speeds available to the solitary structure. These results are qualitatively recovered by our analysis here.

The aim of our investigation is to elucidate the effect of electron superthermality, as manifested through the commonly observed kappa distribution, on the propagation characteristics of nonlinear IA excitations in a simple electronion plasma. We rely on a pseudopotential method to investigate the occurrence and characteristics of arbitrary amplitude IA waves. We shall determine the range of permitted Mach number values for the existence of solitary IA waves in a plasma with excess superthermal electrons, and will, in particular, demonstrate their dependence on "superthermality" (via $\kappa$ ). Recall that the limit $\kappa \rightarrow \infty$ leads to the Maxwellian case, so that the known Mach number domain $[1,1.58]$ (Ref. 23) is recovered in this case (see Fig. 1).

The layout of the paper is as follows. The analytical model equations are presented in Sec. II. In Sec. III, we develop a pseudopotential theory and determine the range of permitted velocity values for the existence of solitary structures. We proceed by numerically evaluating and discussing the propagation velocity range and the effects of superthermality in Secs. IV and V, respectively. Our results are then summarized in Sec. VI. 


\section{GOVERNING MODEL EQUATIONS}

We consider a two-component plasma consisting of

- cold ions (charge $q_{i}=+Z e$, mass $m_{i}$ ), described by the fluid-moment equations, and

- electrons $\left(q_{e}=-e\right.$, mass $\left.m_{e}\right)$, assumed to obey a kappa velocity distribution.

The fluid equations for the ions (in the absence of pressure effects) read

$$
\begin{aligned}
& \frac{\partial n_{i}}{\partial t}+\frac{\partial\left(n_{i} u_{i}\right)}{\partial x}=0, \\
& \frac{\partial u_{i}}{\partial t}+u_{i} \frac{\partial u_{i}}{\partial x}=-\frac{q_{i}}{m_{i}} \frac{\partial \Phi}{\partial x},
\end{aligned}
$$

and the two fluids are coupled through Poisson's equation,

$$
\frac{\partial^{2} \Phi}{\partial x^{2}}=-4 \pi e\left(n_{i} Z-n_{e}\right),
$$

where $n_{i}, u_{i}$, and $\Phi$ are the ion number density, the ion mean velocity, and the electrostatic potential, respectively. The assumption of charge neutrality at equilibrium yields

$$
n_{i 0} Z-n_{e 0}=0,
$$

where the index " 0 " denotes the unperturbed (equilibrium) number density values.

We adopt a kappa distribution for the electrons, and by integrating over velocity space obtain the electron number density, ${ }^{8}$

$$
n_{e}=n_{e 0}\left[1-\frac{e \Phi}{\left(\kappa-\frac{3}{2}\right) k_{B} T_{e}}\right]^{-\kappa+1 / 2},
$$

where the real parameter $\kappa$ measures the deviation from Maxwellian equilibrium. We stress that the latter is recovered in the limit of infinite $\kappa$ at every step.

Normalizing by appropriate scaling quantities, the number density for the electrons may be written in dimensionless form as

$$
n_{e}=\left(1-\frac{\phi}{\kappa-3 / 2}\right)^{-\kappa+1 / 2} .
$$

The normalized ion continuity and momentum equations, and Poisson's equation are

$$
\begin{aligned}
& \frac{\partial n}{\partial t}+\frac{\partial(n u)}{\partial x}=0, \\
& \frac{\partial u}{\partial t}+u \frac{\partial u}{\partial x}=-\frac{\partial \phi}{\partial x}, \\
& \frac{\partial^{2} \phi}{\partial x^{2}}=-n+\left(1-\frac{\phi}{\kappa-3 / 2}\right)^{-\kappa+1 / 2},
\end{aligned}
$$

where the fluid velocity $u_{i}$, the particle density $n_{i}$, and the electrostatic potential $\Phi$ are scaled as $u=u_{i} / c_{s}, n=n_{i} / n_{i 0}$, and $\phi=\Phi / \Phi_{0}$, respectively. Here, $n_{i 0}$ is the equilibrium ion density. We have made use of the quasineutrality relation (5) above. Space and time variables are scaled by the Debye length $\lambda_{D, e}=\left(k_{B} T_{e} / 4 \pi n_{e 0} e^{2}\right)^{1 / 2}=\left(k_{B} T_{e} / 4 \pi Z n_{i 0} e^{2}\right)^{1 / 2}$, and the inverse ion plasma frequency $\omega_{p, i}^{-1}=\left(4 \pi n_{i 0} Z^{2} e^{2} / m_{i}\right)^{-1 / 2}$. Finally, the potential scale reads $\Phi_{0}=k_{B} T_{e} / e$. The characteristic IA sound speed used for velocity normalization is then $c_{s}$ $\equiv\left(Z k_{B} T_{e} / m_{i}\right)^{1 / 2}$.

However, we should note that this expression for the sound speed is applicable to an electron-ion plasma in which the electron density satisfies a Boltzmann distribution. Debye shielding is altered in a plasma with a $\kappa$ distribution, and thus an effective $\kappa$-dependent Debye length is found. ${ }^{34-37}$ Hence, the true sound speed in the plasma model under consideration, with electron density as given by Eq. (6), is kappa dependent and differs from $c_{s}$, as will be seen later.

We should like to emphasize that the normalization used does not contain $\kappa$ at all, and thus the full dependence on $\kappa$ of all variables is exhibited in the normalized expressions, and will be reflected in the numerical work that follows, as is the case for the true sound speed. We note that we have substituted explicitly for $\theta$ as given in the clarification following Eq. (1), and hence the potential is written in terms of the kinetic temperature $T_{e}$ based on a Maxwellian of equal number density and average kinetic energy. ${ }^{1,16,18,34-36}$

\section{ARBITRARY AMPLITUDE SOLITARY WAVE THEORY}

Anticipating the existence of arbitrary amplitude traveling solitary waves, we assume that all fluid variables in the evolution equations depend on a single variable $\xi=x-M t$ (where $M$ is the Mach number, i.e., the pulse propagation velocity normalized by the sound speed, here taken to be the normalization value, $c_{s}$ ). This is the well-known pseudopotential (so-called "Sagdeev") method, leading to a number of ordinary differential equations in a variable of $\xi$; details can be found, e.g., in Refs. 38 and 39. Using the above transformation in Eqs. (8)-(10), the fluid equations become

$$
\begin{aligned}
& -M \frac{\partial n}{\partial \xi}+\frac{\partial(n u)}{\partial \xi}=0, \\
& -M \frac{\partial u}{\partial \xi}+u \frac{\partial u}{\partial \xi}+\frac{\partial \phi}{\partial \xi}=0, \\
& \frac{\partial^{2} \phi}{\partial \xi^{2}}=-n+\left(1-\frac{\phi}{\kappa-3 / 2}\right)^{-\kappa+1 / 2} .
\end{aligned}
$$

After integrating Eqs. (11) and (12) and applying appropriate boundary conditions for localized perturbations, viz., $n \rightarrow 1, u \rightarrow 0$, and $\phi \rightarrow 0$ at $\xi \rightarrow \pm \infty$, we write

$$
-M n+n u=-M,
$$

i.e.,

$$
u=M\left(1-\frac{1}{n}\right)
$$

and 


$$
-M u+\frac{u^{2}}{2}=-\phi .
$$

From Eqs. (15) and (16), we obtain

$$
n=\frac{1}{\sqrt{1-\frac{2 \phi}{M^{2}}}} .
$$

The reality condition $M^{2} \geq 2 \phi$ is then imposed; note that this requirement of a physically realistic density limits positive potential values only.

Substituting Eq. (17) into Eq. (13), multiplying the resulting equation by $d \phi / d \xi$, integrating, and applying the boundary conditions, $d \phi / d \xi \rightarrow 0$ at $\xi \rightarrow \pm \infty$, we find that Poisson's equation takes the form

$$
\frac{1}{2}\left(\frac{d \phi}{d \xi}\right)^{2}+V(\phi)=0
$$

where the (Sagdeev-type) pseudopotential $V(\phi)$ is given by

$$
V(\phi)=M^{2}\left(1-\sqrt{1-\frac{2 \phi}{M^{2}}}\right)+1-\left(1-\frac{\phi}{\kappa-3 / 2}\right)^{-\kappa+3 / 2} .
$$

Equation (18) can be regarded as the "pseudo-energybalance equation" for an oscillating particle of unit mass, with position $\phi$, time $\xi$, velocity $d \phi / d \xi$, and potential $V(\phi)$. We recall, in view of the forthcoming analysis that the Maxwellian limit $^{23}$ is recovered for $\kappa \rightarrow \infty$.

In order for solitary solutions to exist, the following requirements must be fulfilled:

$V(\phi=0)=d V(\phi) /\left.d \phi\right|_{\phi=0}=0$ (at the origin), which represents the requirement that both the electric field and the charge density be zero far from the localized IA solitary structures, and

(ii) $\quad d^{2} V(\phi) /\left.d \phi^{2}\right|_{\phi=0}<0$ (i.e., $V(\phi)$ has a maximum at the origin) so that the sign of the derivative of the charge density is compatible with the sign of the electric field at large distances. Finally, as imposed by the reality of $\phi$, from Eq. (18),

(iii) $\quad V(\phi)<0$ in the region $0<|\phi|<\left|\phi_{m}\right|$; here $\phi_{m}$ denotes the positive root $\left(\phi_{\max }\right)$, for positive potential excitations (or conversely the negative root $\left(\phi_{\min }\right)$, for negative potential excitations).

\section{A. Soliton existence conditions}

The origin at $\phi=0$ defines the equilibrium state, which should represent a local maximum of $V(\phi)$. From Eq. (19), it is clear that both $V(\phi=0)=0$ and $d V(\phi=0) / d \phi=0$ are satisfied at equilibrium. The requirement

$$
\left.\frac{d^{2} V}{d \phi^{2}}\right|_{\phi=0}=\frac{1}{M^{2}}-1-\frac{1}{\kappa-3 / 2}<0
$$

constitutes the soliton (existence) condition to be fulfilled. The root of $d^{2} V /\left.d \phi^{2}\right|_{\phi=0}$ in terms of the Mach number $M$ defines a critical value as a lower limit for $M$, i.e.,

$$
M_{1} \equiv\left(\frac{\kappa-3 / 2}{\kappa-1 / 2}\right)^{1 / 2} \leq 1
$$

For a fixed value of $\kappa$, soliton solutions may exist only for values of the Mach number satisfying $M>M_{1}$. It may easily be shown ${ }^{36}$ that this $\kappa$-dependent expression for $M_{1}$ is actually the IA speed in a two-component plasma with kappadistributed electrons, normalized with respect to the conventional IA speed, $c_{s} \equiv\left(Z k_{B} T_{e} / m_{i}\right)^{1 / 2}$. Thus the existence condition, $M>M_{1}$ implies, as expected, that for solitary waves to exist, they must be traveling at a speed exceeding the true sound speed. Note that the simple value $M_{1}=1$ is recovered for the limit $\kappa \rightarrow \infty$ (simply implying supersonic excitations for IA waves in $e-i$ plasmas with Maxwellian electrons). It is straightforward to see the influence of excess superthermal electrons (via $\kappa$ ) on this soliton velocity threshold. In particular, $M_{1}$ decreases monotonically with decreasing $\kappa$ from the "conventional" value of $M_{1}=1$ found for large $\kappa$, and as $\kappa \rightarrow 3 / 2, M_{1} \rightarrow 0$.

A second (upper) velocity limit for the existence of positive potential solitons arises from the physical requirement of a real ion number density, as expressed by Eq. (17). For $\phi$ $\rightarrow M^{2} / 2$, the density $n$ becomes infinite (and so would the pressure $\sim n^{\gamma}$ in a warm ion model with polytropic index $\gamma$ ). Accessible values of the Mach number are those for which the Sagdeev well yields a root $\phi_{m}$ before this infinite compression limit is reached, and hence we find the largest possible value of $M$ by imposing the requirement $V\left(\phi=M^{2} / 2\right)$ $\geq 0$. The upper limit on the speed of the solitary waves (say, $M_{2}$ ), expressed in terms of the Mach number, is thus obtained by solving the associated equation,

$$
M_{2}^{2}+1-\left(1-\frac{M_{2}^{2}}{2 \kappa-3}\right)^{-\kappa+3 / 2}=0,
$$

for $M_{2}$. As $\kappa \rightarrow \infty$, the last term tends to an exponential form, and hence the upper Mach number limit will then take on the conventional value of 1.58. At the opposite extreme, it can easily be shown that as $\kappa \rightarrow 3 / 2, M_{2} \rightarrow 0$.

Summarizing, assuming the kappa-dependent electron density function given by Eq. (6), positive potential solitary wave solutions of the ion fluid system of equations exist for values of the Mach number $M$ in the range $M_{1}<M<M_{2}$. Clearly, both of these limits vary with kappa, and we need to investigate their dependence on physical parameters.

Relying on the analytical toolbox outlined above, we have performed a parametric investigation, in order to study the properties of arbitrary amplitude solitary waves, as deduced from the pseudopotential $V(\phi)$ given by Eq. (19). Our findings are presented and discussed in the following.

\section{PROPAGATION VELOCITY OF LOCALIZED EXCITATIONS}

Let us first consider the dependence of the critical Mach number values $M_{1}$ and $M_{2}$ on the presence of excess superthermal electrons (superthermality) via $\kappa$, and hence explore the range of accessible Mach numbers as a function of $\kappa$. For the lower velocity threshold, $M_{1}$, this can be inferred analytically upon simple inspection of Eq. (21), as commented on 
in Sec. III A. Recalling the fact that this soliton existence condition represents the requirement of superacoustic propagation speed (i.e., $M_{1}$ is the true IA speed), we see that the true sound speed in this kappa distribution plasma has a lower value than in a Maxwellian plasma, i.e., an increase in superthermal (and the associated reduction in thermal) electrons causes the linear IA wave to propagate at lower speed. This has been shown in a rigorous manner in Ref. 36 .

Unlike the lower limit, the variation of the upper velocity limit imposed by infinite compression of the ions, viz., $M_{2}$, the root of Eq. (22), can only be studied numerically. It is found that $M_{2}$ decreases monotonically as $\kappa$ decreases. In Fig. 1 we have plotted the lower and upper limits, $M_{1}$ and $M_{2}$, respectively, over the range $3 / 2 \leq \kappa \leq 20$, and hence show the permitted range of Mach numbers, which satisfy $M_{1}<M<M_{2}$ and thus support IA solitons in such kappa plasmas. We see that as $\kappa$ is decreased, the available range of Mach numbers over which positive potential IA solitons may exist is reduced.

A few comments are in order, regarding the physical interpretation of Fig. 1. First we note that both curves show an asymptotic behavior as $\kappa$ is increased. As expected, the two limiting Mach numbers tend to 1 and 1.58, respectively, as is well known for the Maxwell-Boltzmann case. ${ }^{23,24}$ That these values are already closely approached from $\kappa \simeq 10$ agrees with earlier studies, where linear wave behavior in plasmas with values of $\kappa$ above $\approx 10$ (roughly) was found to be practically equivalent to that in a Maxwellian plasma. ${ }^{19}$

In this figure, one sees that as expected, both the lower and the upper Mach number limits tend to zero as $\kappa \rightarrow 3 / 2$, the limiting value of $\kappa$. Recalling that a decrease in $\kappa$ measures the deviation from the Maxwellian behavior through an increase in the superthermal electron component and a concomitant decrease in the thermal part of the electron velocity distribution function, we note that higher superthermality results in the shrinking of the permitted region for soliton velocities, compared to what is found for a Maxwellian plasma.

\section{ROLE OF SUPERTHERMALITY}

We wish to study the effect of superthermality on the solitary wave characteristics. First, we consider two values of $\kappa$, viz., $\kappa=16$, which is pseudo-Maxwellian (Fig. 2), and $\kappa$ $=4$, which is strongly non-Maxwellian, with a large superthermal component, and has been found to occur in space plasmas (Fig. 3). In each case we present Sagdeev potential plots which represent positive potential solitary wave structures, calculated for a range of values of the Mach number, $M$, lying in the range, $M_{1}<M<M_{2}$. The amplitude of the solitary electrostatic potential structures (measured by the magnitude of the root, $\phi_{m}$ ) is seen in both figures to increase monotonically as the Mach number is increased, thus showing that behavior of this kind, known for the MaxwellBoltzmann case, applies to low kappa also. Specifically, as $M$ is increased from its lowest value to the largest soliton propagation speed plotted, the normalized electrostatic potential amplitude increases from effectively zero to $\simeq 1.13$ for the pseudo-Maxwellian case (Fig. 2), but to a somewhat lower value, viz., $\simeq 0.77$ for the low- $\kappa$ case (Fig. 3 ). These

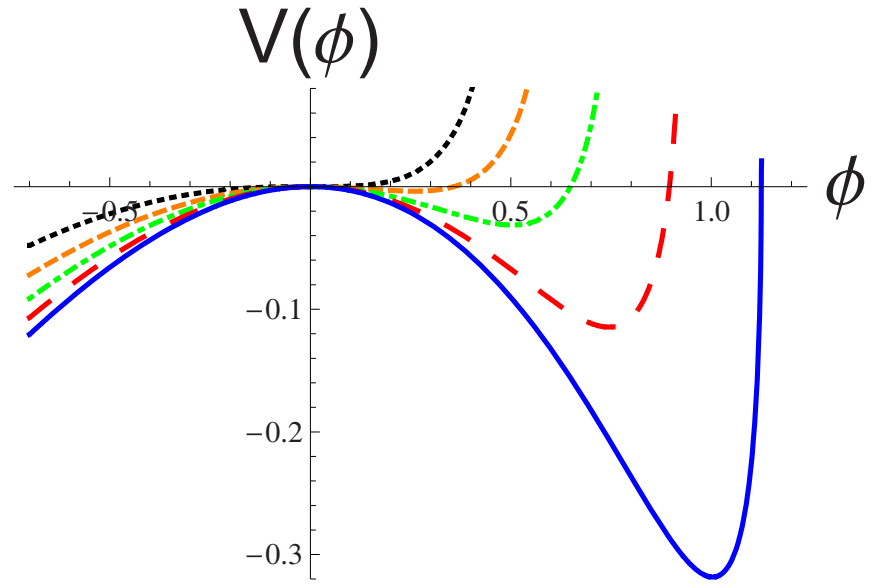

FIG. 2. (Color online) Variation of $V(\phi)$ for $\kappa=16$ and different values of Mach number, $M$. From top to bottom: Dotted curve: $M=0.97$; dashed curve: $M=1.10$; dotted-dashed curve: $M=1.23$; long-dashed curve: $M$ $=1.36$; and solid curve: $M=1.50$.

figures thus indicate that the presence of additional superthermal particles appears to reduce the maximum soliton amplitude.

We also see that in both figures the well depth of the Sagdeev potential curve increases monotonically and dramatically, as the Mach number is increased from close to the lower limit to just below the upper limit. Whereas for the pseudo-Maxwellian case the maximum well depth reaches a normalized value of 0.3 , in the presence of stronger superthermality it is reduced to $\simeq 0.2$. The actual numbers involved in this well depth have less physical significance than the changes in well depth. It follows from Eq. (18) that the well depth is proportional to the square of the maximum electric field, i.e., it is related to the maximum slope of the electrostatic potential profile representing the solitary wave structure. From these two figures we thus deduce that the addition of superthermal particles associated with a lower value of $\kappa$ gives rise to a reduction in the steepness of the

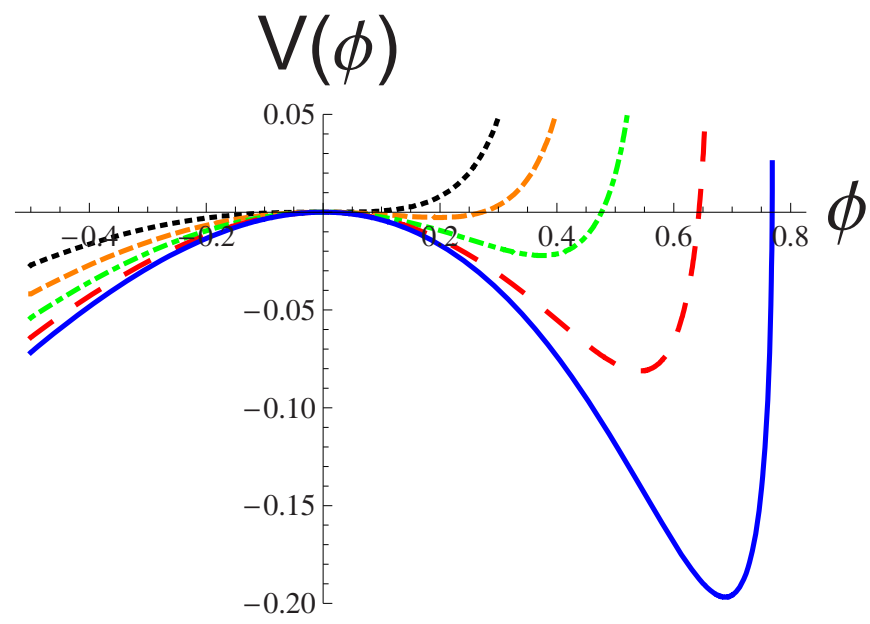

FIG. 3. (Color online) Variation of $V(\phi)$ for $\kappa=4$ and different values of Mach number, $M$. From top to bottom: Dotted curve: $M=0.85$; dashed curve: $M=0.95$; dotted-dashed curve: $M=1.05$; long-dashed curve: $M$ $=1.15$; and solid curve: $M=1.24$. 


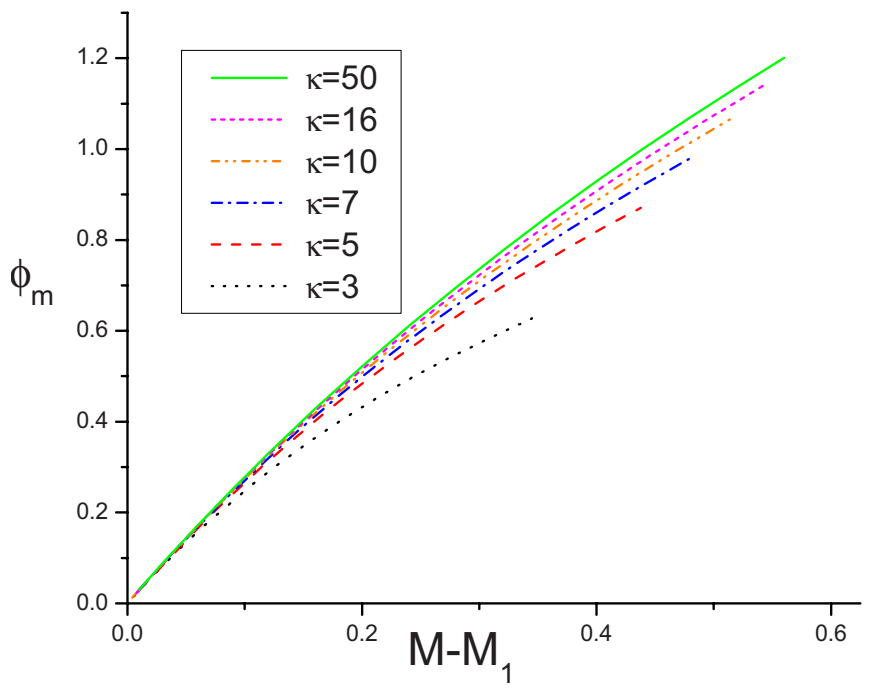

FIG. 4. (Color online) Variation of $\phi_{m}$ with $M-M_{1}$ for different values of $\kappa$. The dotted curve corresponds to $\kappa=3$, the dashed curve to $\kappa=5$, the dotteddashed curve to $\kappa=7$, the dotted-dotted dashed curve to $\kappa=10$, the shortdashed curve to $\kappa=16$, and the solid curve to $\kappa=50$.

soliton profile. These aspects are explored further in Figs. 4 and 5 .

In Fig. 4 we present a set of curves that show the solitary wave amplitude as a function of the increment in Mach number over the soliton existence condition, $M_{1}$ (i.e., $M-M_{1}$ ), for a wide range of values of the parameter $\kappa$, running from a true Maxwellian $(\kappa=50)$ to a strongly non-Maxwellian form $(\kappa=3)$. A widely cited qualitative aspect of the weakly supersonic, small-amplitude, Korteweg-de Vries soliton theory is that larger excitations propagate at higher speeds and are narrower (i.e., "taller is faster and thinner"). Considering first the Maxwellian curve, we note that the potential increases monotonically with $M-M_{1}$ from zero up to the ion compression cutoff at $M=1.58\left(M-M_{1}=0.58\right)$ that the rate of increase is effectively linear for smaller amplitude solitons, relatively close to the lower Mach number limit, and

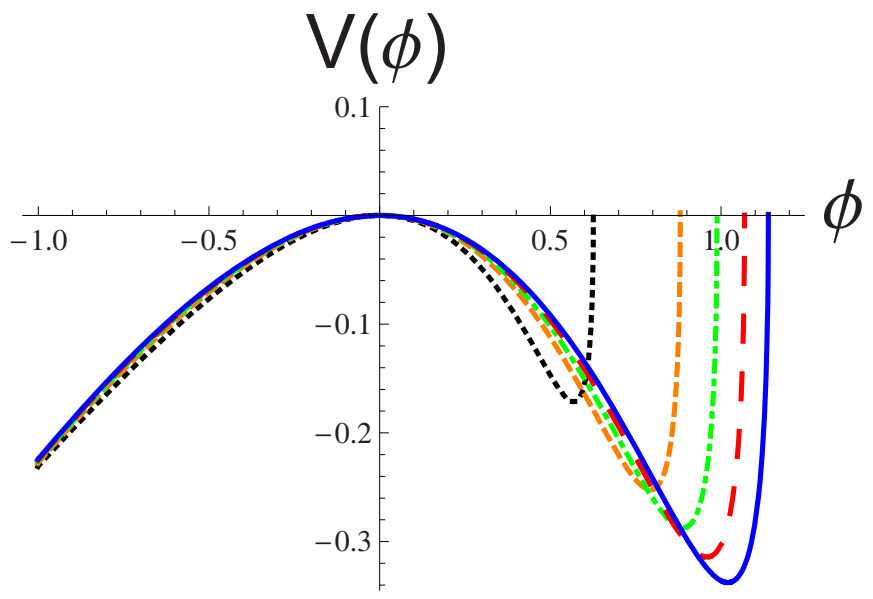

FIG. 5. (Color online) Variation of $V(\phi)$ for different values of $\kappa$, and values of Mach number, $M$, given by $M=M_{2}-0.0015$. The dotted curve corresponds to $\kappa=3$, the dashed curve to $\kappa=5$, the dotted-dashed curve to $\kappa=7$, the long-dashed curve to $\kappa=10$, and the solid curve to $\kappa=16$. that the slope of the curve gradually decreases as the Mach number is increased.

Not only do we see in Fig. 4 that the monotonic behavior is exhibited well beyond the small-amplitude range, but also that it applies whether one has the usual MaxwellBoltzmann plasma or a kappa distribution that is highly nonMaxwellian. For smaller $\kappa$ one finds that at fixed values of $M-M_{1}$ the associated solitary wave potential is lower, i.e., superthermality reduces the amplitude of the solitons. In addition, as we have already seen, the upper Mach number cutoff, $M_{2}$, decreases with increasing superthermality (decreasing $\kappa$ ) and the accessible range for solitons, $M_{2}-M_{1}$, also decreases. These effects are most dramatic as $\kappa$ is reduced from 5 to 3 .

It should be noted that this monotonic behavior is not found universally for arbitrary amplitude acoustic solitons, but appears to relate specifically to solitary waves whose existence domain is restricted by the linear wave speed and an infinite compression or rarefaction, as is the case here. It has, for instance, been observed that in some cases where the upper cutoff in the existence domain arises from the existence of a double layer, the amplitude does not increase monotonically over the full range of accessible Mach numbers. ${ }^{29,40}$

In Fig. 5 we have plotted Sagdeev potential curves for a set of values of $\kappa$ in the range $(16,3)$, i.e., scanning the range from effectively Maxwellian to strongly non-Maxwellian, but this time choosing Mach numbers that are very close to (in fact, within 0.0015 of) the relevant upper limit, $M_{2}$, for the value of $\kappa$ under consideration. As the pseudopotential curve breaks down (ends) at the upper cutoff, the curves in this figure only just cross the axis (yield a root), and as a result graphical representation clearly showing the root is difficult. It will be noted that some of the curves obviously cross the axis, while the others "touch" the axis. Careful numerical evaluation confirms that they too do yield roots. Bearing in mind the fact that we have seen that the amplitude increases monotonically with $M$ at fixed $\kappa$, it follows that we are effectively exploring the largest soliton amplitudes that can be supported by a plasma with a given value of $\kappa$. As found in Fig. 4, we observe that as the superthermal component increases with decreasing $\kappa$ from the pseudoMaxwellian case $(\kappa=16)$, the largest soliton amplitudes that may be achieved decrease monotonically, the normalized potentials dropping from about 1.13 to 0.62 , the value found for $\kappa=3$. The well depth, and thus the steepness of the profile of these "largest" solitons, is also found to fall off monotonically as the superthermal component of the distribution function increases with falling $\kappa$. Having established what occurs when kappa is kept constant, we turn next to a set of calculations for which the Mach number $M$ is kept constant, and $\kappa$ varied.

Figure 6 depicts the variation of the pseudopotential $V(\phi)$ with $\phi$ for fixed Mach number, $M=1.1$, and different values of $\kappa$ ranging from 10 down to 3 . It will be recalled that for $\kappa=10$ we observed an accessible range of Mach numbers that was approaching that for a Maxwellian distribution. We now see that as we introduce a higher proportion of superthermal electrons (i.e., for decreasing $\kappa$ ), the ampli- 


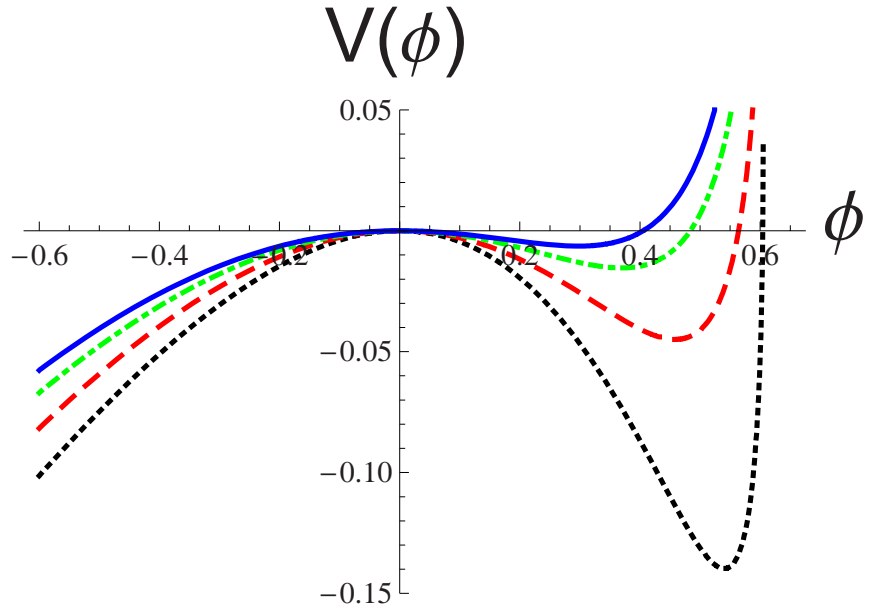

FIG. 6. (Color online) Variation of $V(\phi)$ for fixed $M=1.1$ and different values of $\kappa$. Dotted curve: $\kappa=3$; dashed curve: $\kappa=4$; dotted-dashed curve: $\kappa=6$; and solid curve: $\kappa=10$.

tude of the solitary electrostatic potential structures increases from a normalized value of 0.4 for $\kappa=10$, to 0.6 for $\kappa=3$. This increase in amplitude is indirectly associated with the increase of the superthermal electron component.

We recall that the Mach number is measured relative to a fixed "sound speed," which does not take account of the fact that the true sound speed decreases with decreasing $\kappa$. It is not unusual to normalize with respect to such a fixed characteristic speed when different parameters are being varied. However in this case it follows that as we decrease $\kappa$ at fixed $M$, we are actually increasing the value of $\left(M-M_{1}\right)$. Moreover we have, of course, earlier shown that $\phi_{m}$ increases as $\left(M-M_{1}\right)$ increases. Thus, for fixed $M$, a decrease in $\kappa$ causes an increase in the amount by which the chosen value of Mach number exceeds the local threshold (the true sound speed). Hence it follows that decreasing $\kappa$ yields larger solitons, and as found in this figure, it is thus associated with increasing soliton amplitude over the range $3 \leq \kappa \leq 10$.

We also see that the depth of the Sagdeev pseudopotential well increases dramatically from 0.01 to 0.14 over this range. Thus the maximum slope of the soliton profile increases with decreasing $\kappa$ over this range, i.e., as the superthermal component is increased, the amplitude increases and the soliton profile also becomes steeper.

It is of interest to see whether this effect applies even for very strongly non-Maxwellian plasmas. In Fig. 7, we present some examples of the pseudopotential $V(\phi)$ for a couple of cases with a very strong superthermal component, with $\kappa$ ranging from 1.8 to 2.0 , and $M=0.62$. Such a very hard spectrum, with an extreme accelerated superthermal component, may be found near very strong shocks associated with Fermi acceleration. ${ }^{18}$ We see that both the amplitude of the soliton and the maximum slope of the soliton profile are larger for the lower values (e.g., $\kappa=1.8$ ) than for the higher value, $\kappa=2$. Thus over this narrow range, too, the increase in superthermality gives rise to an increase in soliton amplitude and steepness, at fixed $M$. We have found analogous results even for values of $\kappa$ a little above 1.6.

Finally, we take this discussion a step further with Fig. 8,

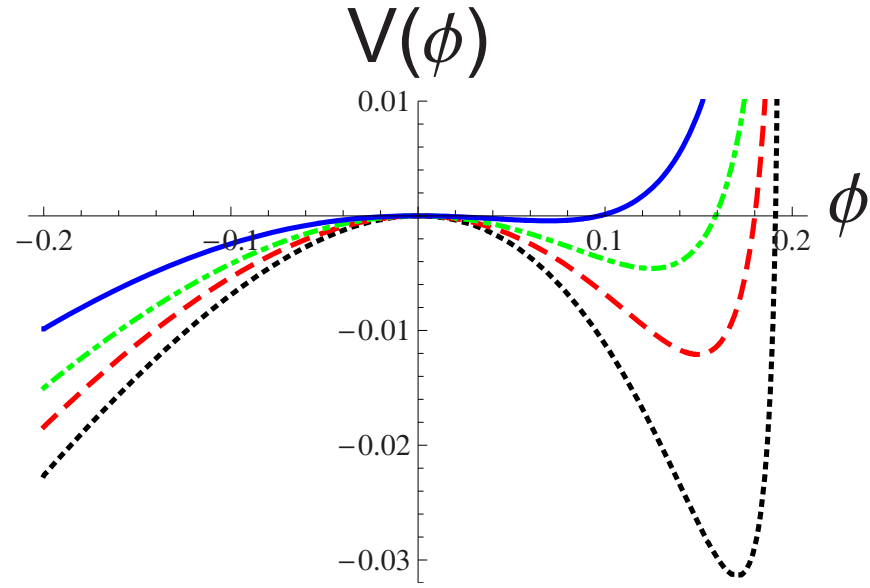

FIG. 7. (Color online) Variation of $V(\phi)$ for fixed $M=0.62$ and different values of $\kappa$. Dotted curve: $\kappa=1.8$; dashed curve: $\kappa=1.85$; dotted-dashed curve: $\kappa=1.9$; and solid curve: $\kappa=2.0$.

showing plots of amplitude against $\kappa$ at four fixed values of Mach number, $M$, ranging from 1.0 to 1.3 . In each case we observe that as $\kappa$ is decreased, the potential at fixed $M$ rises. There are cutoffs at lower values of $\kappa$ for the higher values of $M$, as $M_{2}$ falls below the Mach number under consideration. It is noticeable that the lowest curve, for $M=1.0$, rises much more steeply than the others. This is presumably associated with the rapid fall-off of $M_{1}$ with decreasing $\kappa$ below about 5 , and the resulting rapid rise in $\left(M-M_{1}\right)$ in that range (see Fig. 1), which is associated with a rise in amplitude, $\phi_{m}$ (see Fig. 4).

In summary, the results of our calculations show that the answer to the question of how soliton amplitude varies with $\kappa$ depends significantly on how the question is asked. From Figs. 6-8 one may wish to argue that increased superthermality causes larger amplitude solitons. That is indeed the case at fixed $M$, and thus at increasing values of $\left(M-M_{1}\right)$,

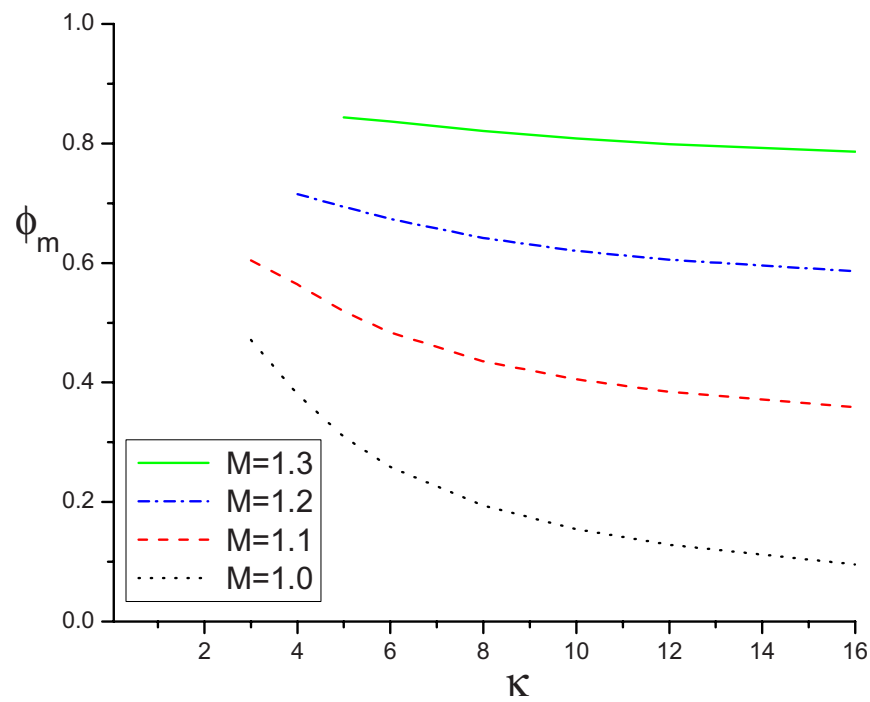

FIG. 8. (Color online) Variation of $\phi_{m}$ with $\kappa$ for different values of the Mach number, $M$. The dotted curve corresponds to $M=1.0$; the dashed curve to $M=1.1$; the dotted-dashed curve to $M=1.2$; and the solid curve to $M=1.3$. 
and its association with increasing $\phi_{m}$. On the other hand, Figs. $2-5$ clearly show that the largest possible values of soliton amplitude for a given value of $\kappa$, attainable for $M$ chosen so that $\left(M_{2}-M\right)$ is small, actually decrease with decreasing values of $\kappa$.

Overall, our results show that superthermality (as measured by the value of the parameter $\kappa$ ) plays a significant role in the modification of solitary electrostatic IA structures and their behavior, but that the resultant behavior depends on the experiment that one is carrying out.

Finally we note that as is the case both for electrons with a Maxwellian distribution and for a Tsallis distribution, only positive solitons have been observed. A wide-ranging numerical search did not reveal any negative potential solitons.

\section{CONCLUSIONS}

In this paper we have studied the existence conditions and the characteristics of IA solitary waves propagating in a plasma composed of cold fluid ions and electrons whose velocity distribution is modeled by a kappa distribution. First, it is noted that only positive potential IA solitary structures are observed in such a plasma, as is the case for a conventional electron-ion plasma in which the electrons are Maxwellian. It thus appears that the presence of additional superthermal particles does not make qualitative changes to this important aspect of soliton behavior, unlike the addition of an additional species, and that the changes are essentially quantitative only, as outlined above. This also agrees with the results obtained for a Tsallis distribution. ${ }^{33}$

Second, the limiting case for the Maxwellian distribution ${ }^{23}$ is recovered for $\kappa \rightarrow \infty$, as expected.

Third, we have shown that the lower Mach number limit for the existence of IA solitons decreases with the presence of a greater superthermal component, i.e., with decreasing $\kappa$. This lower threshold, which tends to zero as $\kappa \rightarrow 3 / 2$, represents the true IA speed in the plasma model under discussion. The upper limit, associated with the ion infinite compression limit, cannot be expressed in a simple closed form, but has to be found numerically. It decreases more rapidly with decreasing $\kappa$ than the lower limit, and hence distributions that may be modeled by lower values of $\kappa$ can support solitons only over a narrower range of accessible Mach numbers. The reduction in accessible solitary wave propagation speeds agrees qualitatively with that found for the case in which the electrons have a Tsallis distribution. ${ }^{33}$

At fixed kappa, that is, for a given velocity distribution function, soliton amplitude and soliton profile steepness both increase monotonically as the Mach number is increased from the threshold value. An interesting result is that the largest possible soliton that can be supported at a fixed value of $\kappa$ is found to decrease as $\kappa$ decreases. This observation is in line with the facts that as $\kappa$ is decreased, the range of available Mach numbers $\left(M_{1}\right.$ to $\left.M_{2}\right)$ decreases, and that soliton amplitude and profile steepness increase monotonically with Mach number [through $\left.\left(M-M_{1}\right)\right]$ at fixed $\kappa$.

On the other hand, for a fixed soliton propagation speed $(M)$ within the accessible range, greater superthermality yields an increase in soliton amplitude, and more pronounced steepness of the soliton profile. This behavior follows because as the threshold Mach number (where the amplitude vanishes) decreases with decreasing $\kappa$, fixed $M$ is increasingly greater than the lower limit, and hence larger soliton amplitudes are generated. These quantitative changes are seen to be particularly important for very low values of $\kappa$, such as $\kappa<4$, i.e., in the presence of a hard spectrum.

Thus, in a plasma in which the electrons have a kappa distribution with lower values of $\kappa$, IA solitons of fixed Mach number have a larger amplitude, and are steeper in their profile, than is the case for conventional solitons occurring in a plasma whose electrons satisfy a Maxwell-Boltzmann velocity distribution. This is because of the increased excess superthermal ("tail") electrons and associated decrease in the thermal component, associated with lower $\kappa$, which give rise to a lower soliton threshold, the true IA speed for the plasma model under discussion. However, the largest possible solitons that may be generated in such a kappa plasma with a specific velocity distribution are found to be smaller than those found in a Maxwell-Boltzmann plasma.

The results reported in this paper may be of importance in the interpretation of localized electrostatic disturbances observed in space plasmas, where $\kappa$ distributions are very common, as well as in laboratory plasmas, in which the presence of an acceleration mechanism may lead to electron velocity distributions that are well modeled by a $\kappa$ distribution.

\section{ACKNOWLEDGMENTS}

Useful discussions with Thomas Baluku, Richard Mace, and Frank Verheest are gratefully acknowledged. The work of N.S.S. and I.K. was supported by a UK EPSRC Science and Innovation award in Plasma Physics (CPP grant EP/ D06337X/1). N.S.S. would like to thank Guru Nanak Dev University, Amritsar, India for providing leave. Part of the work was carried out by I.K. during a research visit to the University of Sydney. I.K. is grateful to the UK Royal Society for the award of a travel grant, and to the University of Sydney for its hospitality and local support provided during that visit. The research is also supported in part by the National Research Foundation of South Africa (NRF). Any opinion, findings, and conclusions or recommendations expressed in this material are those of the authors and therefore the NRF does not accept any liability in regard thereto.

${ }^{1}$ V. M. Vasyliunas, J. Geophys. Res. 73, 2839, DOI:10.1029/ JA073i009p02839 (1968).

${ }^{2}$ M. P. Leubner, J. Geophys. Res. 87, 6335, DOI:10.1029/ JA087iA08p06335 (1982).

${ }^{3}$ T. P. Armstrong, M. T. Paonessa, E. V. Bell, and S. M. Krimigis, J. Geophys. Res. 88, 8893, DOI:10.1029/JA088iA11p08893 (1983).

${ }^{4}$ M. P. Leubner, Phys. Plasmas 11, 1308 (2004).

${ }^{5}$ P. Schippers, M. Blanc, N. André, I. Dandouras, G. R. Lewis, L. K. Gilbert, A. M. Persoon, N. Krupp, D. A. Gurnett, A. J. Coates, S. M. Krimigis, D. T. Young, and M. K. Dougherty, J. Geophys. Res. 113, A07208, DOI:10.1029/2008JA013098 (2008).

${ }^{6}$ M. A. Hellberg, R. L. Mace, R. J. Armstrong, and G. Karlstad, J. Plasma Phys. 64, 433 (2000).

${ }^{7}$ A. Hasegawa, K. Mima, and M. Duong-van, Phys. Rev. Lett. 54, 2608 (1985).

${ }^{8}$ T. K. Baluku and M. A. Hellberg, Phys. Plasmas 15, 123705 (2008).

${ }^{9}$ S. P. Christon, D. G. Mitchell, D. J. Williams, L. A. Frank, C. Y. Huang, 
and T. E. Eastman, J. Geophys. Res. 93, 2562, DOI:10.1029/ JA093iA04p02562 (1988).

${ }^{10}$ W. C. Feldman, R. C. Anderson, J. R. Asbridge, S. J. Bame, J. T. Gosling, and R. D. Zwickl, J. Geophys. Res. 87, 632, DOI:10.1029/ JA087iA02p00632 (1982); W. C. Feldman, R. C. Anderson, S. J. Bame, S. P. Gary, J. T. Gosling, D. J. McComas, M. F. Thomsen, G. Paschmann, and M. M. Hoppe, ibid. 88, 96, DOI:10.1029/JA088iA01p00096 (1983).

${ }^{11}$ V. Pierrard and J. F. Lemaire, J. Geophys. Res. 101, 7923, DOI:10.1029/ 95JA03802 (1996).

${ }^{12}$ M. Maksimovic, V. Pierrard, and J. F. Lemaire, Astron. Astrophys. 324, 725 (1997).

${ }^{13}$ R. A. Treumann, Astrophys. Space Sci. 277, 81 (2001).

${ }^{14}$ R. A. Treumann, C. H. Jaroschek, and M. Scholer, Phys. Plasmas 11, 1317 (2004).

${ }^{15}$ M. R. Collier, Adv. Space Res. 33, 2108 (2004).

${ }^{16}$ D. Summers and R. M. Thorne, Phys. Fluids B 3, 1835 (1991).

${ }^{17}$ H. Abbasi and H. H. Pajouh, Phys. Plasmas 14, 012307 (2007).

${ }^{18}$ R. L. Mace and M. A. Hellberg, Phys. Plasmas 2, 2098 (1995).

${ }^{19}$ M. A. Hellberg and R. L. Mace, Phys. Plasmas 9, 1495 (2002).

${ }^{20}$ A. F. Viñas, R. L. Mace, and R. F. Benson, J. Geophys. Res. 110, A06202, DOI:10.1029/2004JA010967 (2005).

${ }^{21}$ M.-J. Lee, Phys. Plasmas 14, 032112 (2007).

${ }^{22}$ H. Washimi and T. Taniuti, Phys. Rev. Lett. 17, 996 (1966).

${ }^{23}$ R. Z. Sagdeev, Rev. Plasma Phys. 4, 23 (1966).

${ }^{24}$ F. F. Chen, Introduction to Plasma Physics and Controlled Fusion (Plenum, New York, 1984).

${ }^{25}$ M. Y. Yu, P. K. Shukla, and S. Bujarbarua, Phys. Fluids 23, 2146 (1980).
${ }^{26}$ S. Baboolal, R. Bharuthram, and M. A. Hellberg, J. Plasma Phys. 44, 1 (1990).

${ }^{27}$ J. F. McKenzie, T. B. Doyle, M. A. Hellberg, and F. Verheest, J. Plasma Phys. 71, 163 (2005).

${ }^{28}$ J. F. McKenzie, F. Verheest, T. B. Doyle, and M. A. Hellberg, Phys. Plasmas 11, 1762 (2004).

${ }^{29}$ F. Verheest, T. Cattaert, and M. A. Hellberg, Phys. Plasmas 12, 082308 (2005).

${ }^{30}$ J. F. McKenzie, F. Verheest, T. B. Doyle, and M. A. Hellberg, Phys. Plasmas 12, 102305 (2005).

${ }^{31}$ R. Bharuthram and P. K. Shukla, Planet. Space Sci. 40, 973 (1992).

${ }^{32}$ Nonextensive Entropy: Interdisciplinary Applications, edited by M. GellMann and C. Tsallis (Oxford University Press, New York, NY, 2004).

${ }^{33}$ I. D. Dubinova and A. E. Dubinov, Tech. Phys. Lett. 32, 575 (2006).

${ }^{34}$ Y. F. Chateau and N. Meyer-Vernet, J. Geophys. Res. 96, 5825, DOI:10.1029/90JA02565 (1991).

${ }^{35}$ D. A. Bryant, J. Plasma Phys. 56, 87 (1996).

${ }^{36}$ R. L. Mace, M. A. Hellberg, and R. A. Treumann, J. Plasma Phys. 59, 393 (1998).

${ }^{37}$ R. L. Mace, G. Amery, and M. A. Hellberg, Phys. Plasmas 6, 44 (1999).

${ }^{38}$ F. Verheest, M. A. Hellberg, and G. S. Lakhina, Astrophys. Space Sci. Trans. 3, 15 (2007).

${ }^{39}$ F. Verheest, in Aspects of Plasma Physics, edited by P. K. Shukla, B. Eliasson, L. Stenflo, and R. Bingham (World Scientific, Singapore, 2008), p. 316.

${ }^{40}$ F. Verheest, M. A. Hellberg, and I. Kourakis, Phys. Plasmas 15, 112309 (2008). 